\title{
Medical Position Statement on the Diagnosis and Therapy for Anal Fissure
}

On the basis of our experience, we favor the following algorithm for diagnosis and therapy for an anal fissure disorder.

Due to the deficiencies in most clinical trials at hand, it is impossible to deduce evidence-based recommendations for therapy.

We miss a widely recognized definition of chronic anal fissure.

We have found that many authors do not take full account of all of the results of pharmacodynamics with regard to dosage, penetration kinetics, galenic formulation, site of application, and bioavailability of topically applied drugs.

We believe the hypothesis to be questionable at least that hypertonic anal sphincter is responsible for the formation and development of chronic anal fissures.

We therefore have serious doubts about the reasonability to reduce anal sphincter hypertonia either by medication or surgery to heal chronic anal fissures (Table 12.1).

Table 12.1 Algorithm for anal fissure diagnosis and treatment

\begin{tabular}{|c|c|c|c|c|}
\hline Etiopathogenesis & \multicolumn{4}{|c|}{ Infection, trauma, reduced perfusion } \\
\hline Symptoms & \multicolumn{4}{|c|}{ Pain with defecation, blood on stool and/or toilet paper } \\
\hline Diagnosis & \multicolumn{2}{|c|}{$\begin{array}{l}\text { Inspection, palpation, } \\
\text { speculum, } \\
\text { proctoscopy, probe if } \\
\text { necessary }\end{array}$} & \multicolumn{2}{|c|}{$\begin{array}{l}\text { DD: secondary fissures, erosions, } \\
\text { rhagades, } \\
\text { anal cancer, anal pain syndromes }\end{array}$} \\
\hline Findings & \multicolumn{2}{|c|}{ Acute anal fissure (superficial) } & \multicolumn{2}{|c|}{$\begin{array}{l}\text { Chronic anal fissure without or with } \\
\text { secondary } \\
\text { changes (skin tag, anal papilla, cryptitis, } \\
\text { fistula) }\end{array}$} \\
\hline Basic therapy & \multicolumn{2}{|c|}{$\begin{array}{l}\text { Dietary fiber, no laxatives, } \\
\text { sufficient liquid intake, } \\
\text { warm sitz baths }\end{array}$} & \multicolumn{2}{|c|}{$\begin{array}{l}\text { Dietary fiber, no laxatives, } \\
\text { sufficient liquid intake, } \\
\text { warm sitz baths }\end{array}$} \\
\hline Therapy & \multicolumn{2}{|c|}{$\begin{array}{l}\text { Self-bougienage and/or } \\
\text { diltiazem cream }\end{array}$} & \multicolumn{2}{|c|}{ Self-bougienage and/or diltiazem cream } \\
\hline Development & Healing & Persistence & Healing & Persistence \\
\hline $\begin{array}{l}\text { Further therapy in } \\
\text { case of persistence }\end{array}$ & & Fissurectomy & & $\begin{array}{l}\text { Fissurectomy }+ \text { excision of } \\
\text { secondary changes }\end{array}$ \\
\hline Recurrence & \multicolumn{2}{|c|}{ Conservative or fissurectomy } & \multicolumn{2}{|c|}{ Conservative or fissurectomy } \\
\hline
\end{tabular}

